\title{
Impact of frequent cerebrospinal fluid sampling on $A \beta$ levels: systematic approach to elucidate influencing factors
}

Bianca Van Broeck', Maarten Timmers ${ }^{1,5}$, Steven Ramael ${ }^{2}$, Jennifer Bogert ${ }^{3}$, Leslie M. Shaw ${ }^{4}$, Marc Mercken $^{1}$, John Slemmon ${ }^{3}$, Luc Van Nueten', Sebastiaan Engelborghs ${ }^{5,6}$ and Johannes Rolf Streffer ${ }^{1,5^{*}}$

\begin{abstract}
Background: Cerebrospinal fluid (CSF) amyloid-beta (A $\beta$ ) peptides are predictive biomarkers for Alzheimer's disease and are proposed as pharmacodynamic markers for amyloid-lowering therapies. However, frequent sampling results in fluctuating CSF A $\beta$ levels that have a tendency to increase compared with baseline. The impact of sampling frequency, volume, catheterization procedure, and ibuprofen pretreatment on CSF A $\beta$ levels using continuous sampling over $36 \mathrm{~h}$ was assessed.

Methods: In this open-label biomarker study, healthy participants ( $n=18$; either sex, age 55 - 85 years) were randomized into one of three cohorts ( $n=6 /$ cohort; high-frequency sampling). In all cohorts except cohort 2 (sampling started $6 \mathrm{~h}$ post catheterization), sampling through lumbar catheterization started immediately post catheterization. Cohort 3 received ibuprofen $(800 \mathrm{mg}$ ) before catheterization. Following interim data review, an additional cohort $4(n=6)$ with an optimized sampling scheme (low-frequency and lower volume) was included. CSF $A \beta_{1-37}, A \beta_{1-38}, A \beta_{1-40}$, and $A \beta_{1-42}$ levels were analyzed.

Results: Increases and fluctuations in mean CSF A $\beta$ levels occurred in cohorts 1-3 at times of high-frequency sampling. Some outliers were observed (cohorts 2 and 3) with an extreme pronunciation of this effect. Cohort 4 demonstrated minimal fluctuation of CSF A $\beta$ both on a group and an individual level. Intersubject variability in CSF $A \beta$ profiles over time was observed in all cohorts.

Conclusions: CSF A $\beta$ level fluctuation upon catheterization primarily depends on the sampling frequency and volume, but not on the catheterization procedure or inflammatory reaction. An optimized low-frequency sampling protocol minimizes or eliminates fluctuation of CSF A $\beta$ levels, which will improve the capability of accurately measuring the pharmacodynamic read-out for amyloid-lowering therapies.
\end{abstract}

Trial registration: ClinicalTrials.gov NCT01436188. Registered 15 September 2011.

Keywords: A $\beta$ peptides, Alzheimer's disease, Catheterization, Cerebrospinal fluid, Sampling frequency

\section{Background}

Alzheimer's disease (AD) neuropathology is characterized by deposition in the brain of amyloid plaques, consisting mainly of amyloid-beta $(A \beta)$ peptides, and neurofibrillary tangles, composed of hyperphosphorylated tau protein.

\footnotetext{
* Correspondence: jstreffe@its.jnj.com

Bianca Van Broeck and Maarten Timmers are joint first authors.

'Janssen Research \& Development, a division of Janssen Pharmaceutica N.V., Beerse, Belgium

${ }^{5}$ Reference Center for Biological Markers of Dementia (BIODEM), Institute Born-Bunge, University of Antwerp, Antwerp, Belgium

Full list of author information is available at the end of the article
}

Levels of cerebrospinal fluid (CSF) biomarkers $A \beta$ and tau closely reflect the central pathogenic processes in $A D$ and have proven their utility in evaluating disease risk or prognosis, guiding clinical diagnosis and monitoring therapeutic interventions $[1,2]$.

$A \beta$ peptides are proteolytic cleavage products of amyloid precursor protein, formed by $\beta$ - and $\gamma$-secretase activity. $A \beta$ species predominantly include peptides of $1-$ 40 amino acids $\left(\mathrm{A} \beta_{1-40}\right)$ and $1-42$ amino acids $\left(\mathrm{A} \beta_{1-42}\right)$. Several studies report pronounced increases in CSF A $\beta$ levels relative to baseline upon repeated CSF sampling with spinal catheters [3-9]. Additionally, methodological 
challenges in measuring A $\beta$ in CSF are well described, but influencing factors are only partly understood [1013].

Considering these challenges, there is a clear need to optimize and standardize the technique of continuous CSF sampling and measurement of CSF A $\beta$ for application in clinical trials for $A \beta$-lowering compounds. We performed a study utilizing continuous CSF sampling with indwelling catheters over $36 \mathrm{~h}$ in healthy older participants to evaluate the effects on CSF A $\beta$ levels when applying various sampling protocols. We investigated the impact of sampling frequency, volume, catheterization procedure, and pretreatment with an anti-inflammatory agent (ibuprofen) on changes in CSF $A \beta$ levels over time. We also monitored the impact of different sampling schemes and CSF volume on the safety and tolerability of procedures.

\section{Methods}

\section{Study population}

Healthy men or women ( $n=24$; age $55-85$ years, body mass index (BMI) $18-35 \mathrm{~kg} / \mathrm{m}^{2}$ ) with Mini-Mental State Examination (MMSE) scores $\geq 27$ were enrolled. None of these participants had any significant history of or clinically significant current medical illness at screening or admission, and neither did they have any sign of intracranial pressure as confirmed by fundoscopy.

Participants were excluded if they received the following drugs: aspirin (even low dose) within 5 days, low molecular weight heparin within $12 \mathrm{~h}$, or anticoagulant treatment (besides the heparin already described) within
1 week before spinal catheterization (Additional file 1: Inclusion and exclusion criteria).

The study was conducted in accordance with the ethical principles that have their origin in the Declaration of Helsinki and that are consistent with current International Conference on Harmonization (ICH) guidelines on good clinical practices (GCP) and applicable regulatory requirements, and in compliance with the study protocol. The study protocol was reviewed and approved by the Institutional Review Board (Commissie voor Medische Ethiek, ZNA, Antwerp, Belgium). Written informed consent was obtained for participation and a separate consent for pharmacogenomic (DNA) sampling.

\section{Study design}

This open-label, single-center (Belgium), biomarker study (ClinicalTrials.gov NCT01436188) without investigational medicinal product, conducted between September 2011 and June 2013, consisted of a screening period (between 21 and 2 days before catheter insertion), an in-patient CSF collection period (1-3 days), and a follow-up period ( 714 days after removal of the catheter). For each participant, the maximal study duration did not exceed 6 weeks (Fig. 1).

Initially participants $(n=18)$ were randomized into one of three cohorts $(n=6 /$ cohort): cohort 1 , highfrequency CSF sampling scheme with immediate sampling post catheterization; cohort 2, high-frequency CSF sampling scheme with delayed sampling, starting $6 \mathrm{~h}$ post catheterization; and cohort 3, high-frequency CSF

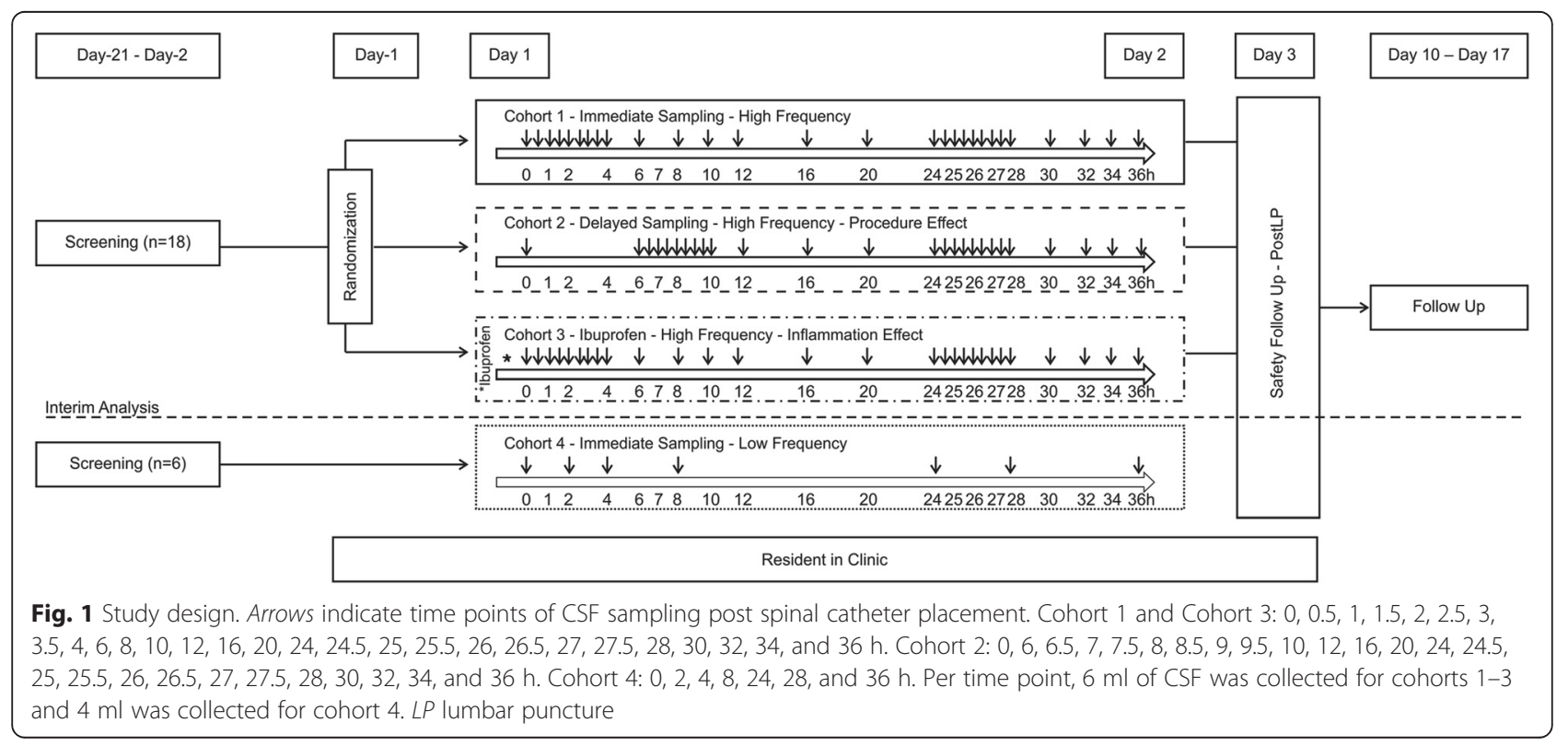


sampling scheme with immediate sampling and prelumbar puncture treatment with ibuprofen on day 1 (single dose, $800 \mathrm{mg} 2 \mathrm{~h}$ before catheterization). CSF was sampled at the following time points post spinal catheter placement for cohorts 1 and 3: $0,0.5,1,1.5,2,2.5,3$, $3.5,4,6,8,10,12,16,20,24,24.5,25,25.5,26,26.5,27$, $27.5,28,30,32,34$, and $36 \mathrm{~h}$. For cohort 2, the sampling time points were as follows: $0,6,6.5,7,7.5,8,8.5,9,9.5$, $10,12,16,20,24,24.5,25,25.5,26,26.5,27,27.5,28$, 30, 32, 34, and $36 \mathrm{~h}$.

Following an interim review of the data from cohorts $1-3$, six healthy older participants were allocated to an additional cohort: cohort 4, low-frequency CSF sampling scheme with immediate sampling. CSF was sampled at the following time points post spinal catheter placement for cohort 4: 0, 2, 4, 8, 24, 28, and 36 h (Fig. 1).

\section{CSF sampling}

CSF samples (6 $\mathrm{ml}$ for cohorts $1-3$ and $4 \mathrm{ml}$ for cohort 4 , per time point) were collected over $36 \mathrm{~h}$ using a lumbar spinal indwelling CSF catheter. On day 1, all participants received a lumbar indwelling CSF catheter (interspace of L3 and L4 or L4 and L5 of the lumbar spine) between 6:00 and 9:00 a.m. The introducer needle (Tuohy needle) with stylet in place was inserted at the superior aspect of the inferior spinous process in the midline and approximately $15^{\circ}$ cephalad. The stylet was removed and the peridural space was entered using a loss of resistance technique. A Spinocath Catheter (22 Gauge; BBraun Melsungen AG, Germany) with a spinal needle inside was introduced through the Tuohy needle to perforate the dura mater ("dural pop"). The spinal needle was withdrawn while moving up the spinal catheter into the subarachnoid space for $10-15 \mathrm{~cm}$ (distance tip to skin). The Tuohy needle was subsequently removed. To reduce the risk of CSF leakage, participants were preferably placed on a bed, resting in a supine position during sampling and for up to $12-24 \mathrm{~h}$ after removal of the catheter. No specific cognitive activity was mandated, but most participants were reading or listening to music.

CSF samples were collected with a syringe (BD Plastipack 2 and $5 \mathrm{ml}$ ) under sterile conditions from the moment of catheter placement ( $0 \mathrm{~h}$ or baseline sample) through the 36 -h assessment period. Samples were collected in $10 \mathrm{ml}$ (catalog number 62.610.018)/12 ml (catalogue number 60.541.004) Sarstedt Liquor tubes, which were immediately placed on melting ice and gently inverted for adequate mixing. The collected CSF volume was aliquoted by immediate transfer of $500 \mu \mathrm{l}$ samples to multiple storage tubes (Micronic $1.4 \mathrm{ml}$ noncoded $\mathrm{U}$ bottom tubes in Comorack-96 (catalogue number MP22502) with caps from FluidX (Split TPE Capcluster Blue, catalogue number $65-53028)$ ) and stored at $-70{ }^{\circ} \mathrm{C}$.
All sampling and processing materials used were analyzed for their $A \beta$ adsorption capacity and were found acceptable $(<20 \%)$ for avoiding any major influence on the $A \beta$ read-outs (unpublished data).

\section{Analysis of CSF $A \beta$ levels}

A qualified, specific, and sensitive prototype multiplex immunoassay developed by Janssen Research \& Development and based on Meso Scale Discovery (MSD) electrochemiluminescence detection technology was utilized for simultaneous detection of four CSF $A \beta$ species $\left(A \beta_{1-37}, A \beta_{1-38}\right.$, $\mathrm{A} \beta_{1-40}$, and $\left.\mathrm{A} \beta_{1-42}\right)[14,15]$. Purified monoclonal antibodies specific for $A \beta x-37$ (JRD/A $337 / 3$ ), $A \beta x-38$ (J\&JPRD/ $\mathrm{A} \beta 38 / 5), \mathrm{A} \beta \mathrm{x}-40$ (JRF/cA $\beta 40 / 28)$, and $\mathrm{A} \beta \mathrm{x}-42$ (JRF/cA $\beta 42 /$ 26) were coated on MSD 4-plex 96-well plates as capture antibodies. JRF/A $\beta N / 25$, which displays specificity for $A \beta$ isoforms with intact $\mathrm{N}$-terminus (i.e., full-length $A \beta$ ), was utilized as the detection antibody [16]. The CSF $A \beta_{1-37}$, $A \beta_{1-38}, A \beta_{1-40}$, and $A \beta_{1-42}$ concentrations were determined using a standard curve with a four-parameter logistic model with $1 / Y^{2}$ weighting function.

One operator performed all of the experimental analyses for participants in cohorts $1-3$, in a random fashion over 3 consecutive days. The experimental analysis for cohort 4 was performed by one operator on the same day. All samples from each participant were analyzed in duplicate on the same plate. Only mean values with a replicate well coefficient of variation $(\mathrm{CV}) \leq 20 \%$ were accepted.

\section{Analysis of baseline CSF $A \beta_{1-42}$, T-tau, and phosphorylated tau $_{181 \mathrm{P}}$ levels}

Baseline $\mathrm{A} \beta_{1-42}, \mathrm{P}-\mathrm{tau}_{181 \mathrm{~B}}$, and total tau (T-tau) concentrations (i.e., directly after catheter insertion) were measured utilizing INNO-BIA AlzBio3 kit reagents and the Luminex analytical platform [17]. Diagnostic threshold CSF concentrations for AD versus normal controls for $\mathrm{A} \beta_{1-42}$ were applied to the current sample set to judge the likelihood of having cerebral amyloid plaque deposition [18].

\section{Apolipoprotein E (APOE) epsilon 4 carrier status}

Blood samples $(10 \mathrm{ml})$ were collected in tubes containing potassium/sodium EDTA. DNA was isolated using Puregene chemistry and automated extraction using an Autopure LS. The aliquots of DNA samples from all participants were genotyped in a multiplex reaction using PCR/ligation detection reaction [19].

\section{Safety assessments}

Adverse events (AEs) were monitored. Laboratory tests, examination of vital signs, resting 12-lead ECGs, physical and neurological examinations, and fundoscopy were performed. 


\section{Sample size}

Based on CSF determinations of $A \beta_{1-42}, A \beta_{1-40}$, and $\mathrm{A} \beta_{1-38}$ with a similar enzyme-linked immunosorbent assay (ELISA) method (Janssen, unpublished data), the $\mathrm{CV}$ of percentage change from baseline was estimated to be $\sim 13 \%$. A sample size of five completers per group was expected to produce two-sided $95 \%$ confidence intervals with $16 \%$ precision compared with natural fluctuation of around $15 \%$ in the reference study (ClinicalTrials.gov NCT01556217) and was considered reasonable for precise estimation of mean.

\section{Results}

Demographics, baseline characteristics, and AD biomarker pattern

Twenty-four participants were enrolled, most were men with mean age of 64 years. The average measures for age, weight, height, BMI, and MMSE score at screening were comparable among cohorts $(p=0.42,0.16,0.56$, 0.66 , and 0.45 , respectively, $F$ test) (Table 1 ). AD biomarkers were analyzed. Two participants had $\mathrm{A} \beta_{1-42}$ below the threshold concentration $(\leq 249 \mathrm{pg} / \mathrm{ml})$ [18], suggested to be pathologic, but none had elevated T-tau or P-tau $\tan _{181}$ values (Additional file 2: Figure S1). Hence, no participant had a typical AD biomarker pattern. All participants completed the study.

\section{CSF $A \beta$ levels}

Cohort 1: high-frequency CSF sampling scheme with immediate sampling

Mean CSF $A \beta_{1-40}$ levels fluctuated with a tendency for increasing levels relative to baseline over the sampling period (Fig. 2). CSF A $\beta$ levels were particularly increased during periods of frequent sampling (sampling every 30 min: $0-4 \mathrm{~h}$ and $24-28 \mathrm{~h}$ ). After the $12-\mathrm{h}$ time point, two participants showed increases in CSF $A \beta_{1-40}$ levels of $>20 \%$ at several time points compared with baseline. Other participants had a rather stable pattern of CSF A $\beta$ over the $36 \mathrm{~h}$ (Fig. 3a).

\section{Cohort 2: high-frequency CSF sampling scheme with de- layed sampling}

In contrast to cohort 1 , mean CSF $A \beta_{1-40}$ levels at baseline and $6 \mathrm{~h}$ post catheterization were similar in cohort 2 (Fig. 2). CSF A $\beta$ levels increased in periods of frequent sampling (sampling every $30 \mathrm{~min}$ : 6-10 $\mathrm{h}$ and $24-28 \mathrm{~h}$ ). Notably in cohort 2, unlike cohort 1 , CSF A $\beta$ levels remained elevated after the first period of frequent

Table 1 Demographics and baseline characteristics of study participants by cohort (all randomized participants)

\begin{tabular}{|c|c|c|c|c|c|}
\hline & $\begin{array}{l}\text { Cohort } 1 \\
(n=6)\end{array}$ & $\begin{array}{l}\text { Cohort } 2 \\
(n=6)\end{array}$ & $\begin{array}{l}\text { Cohort } 3 \\
(n=6)\end{array}$ & $\begin{array}{l}\text { Cohort } 4 \\
(n=6)\end{array}$ & $\begin{array}{l}\text { Total } \\
(N=24)\end{array}$ \\
\hline Age (years) & $62(4.3)$ & $64(6.4)$ & $66(1.7)$ & $63(3.3)$ & $64(4.3)$ \\
\hline Sex (men), $n(\%)$ & $6(100.0)$ & $5(83.3)$ & $6(100.0)$ & $5(83.3)$ & $22(91.7)$ \\
\hline \multicolumn{6}{|l|}{ Race, $n(\%)$} \\
\hline White & $6(100.0)$ & $6(100.0)$ & $5(83.3)$ & $6(100.0)$ & $23(95.8)$ \\
\hline Black or African American & 0 & 0 & $1(16.7)$ & 0 & $1(4.2)$ \\
\hline \multicolumn{6}{|l|}{ Ethnicity, n (\%) } \\
\hline Not Hispanic or Latino & $6(100.0)$ & $6(100.0)$ & $6(100.0)$ & $6(100.0)$ & $24(100.0)$ \\
\hline Weight (kg) & $83(10.8)$ & $72(8.3)$ & $77(5.6)$ & 79 (10.6) & $78(9.6)$ \\
\hline Height (cm) & $176(7.8)$ & $170(9.5)$ & $171(9.2)$ & $173(7.6)$ & $172(8.4)$ \\
\hline $\mathrm{BMI}\left(\mathrm{kg} / \mathrm{m}^{2}\right)$ & $27.1(3.8)$ & $25(2.3)$ & $26.6(2.7)$ & $26.6(3.5)$ & $26.3(3.0)$ \\
\hline Total MMSE at screening & $29.2(0.8)$ & $29.0(1.3)$ & $29.7(0.5)$ & $29.7(0.8)$ & $29.4(0.9)$ \\
\hline$A \beta_{1-42}(p g / m l)$ & $393.2(26.4)$ & $380.6(44.1)$ & $326.1(75.4)$ & $319.2(97.6)$ & $354.8(70.6)$ \\
\hline T-tau (pg/ml) & $74.5(20.0)$ & $62.7(28.9)$ & 46.7 (20.6) & 50.7 (21.9) & $58.6(24.3)$ \\
\hline P-tau $181 P(p g / m l)$ & $30.7(8.2)$ & $22.2(4.9)$ & $20.4(6.9)$ & $24.9(13.0)$ & $24.6(9.1)$ \\
\hline \multicolumn{6}{|l|}{$A P O E \varepsilon 4$ carrier } \\
\hline Yes & $1(16.7)$ & $1(16.7)$ & 0 & $2(33.3)$ & $4(16.7)$ \\
\hline No & $4(66.7)$ & $3(50.0)$ & $5(83.3)$ & $4(66.7)$ & $16(66.7)$ \\
\hline Unknown & $1(16.7)$ & $2(33.3)$ & $1(16.7)$ & 0 & $4(16.7)$ \\
\hline
\end{tabular}

Data shown as mean (standard deviation), unless otherwise specified

Cohort 1: immediate sampling, high frequency; cohort 2: delayed sampling, high frequency, procedure effect; cohort 3: ibuprofen, high frequency, inflammation effect; cohort 4: immediate sampling, low frequency. APOE, apolipoprotein $\mathrm{E}$ gene; $B M I$, body mass index; $M M S E$, mini-mental state examination; $A \beta$, amyloid beta; P-tau, phosphorylated tau; T-tau, total tau 


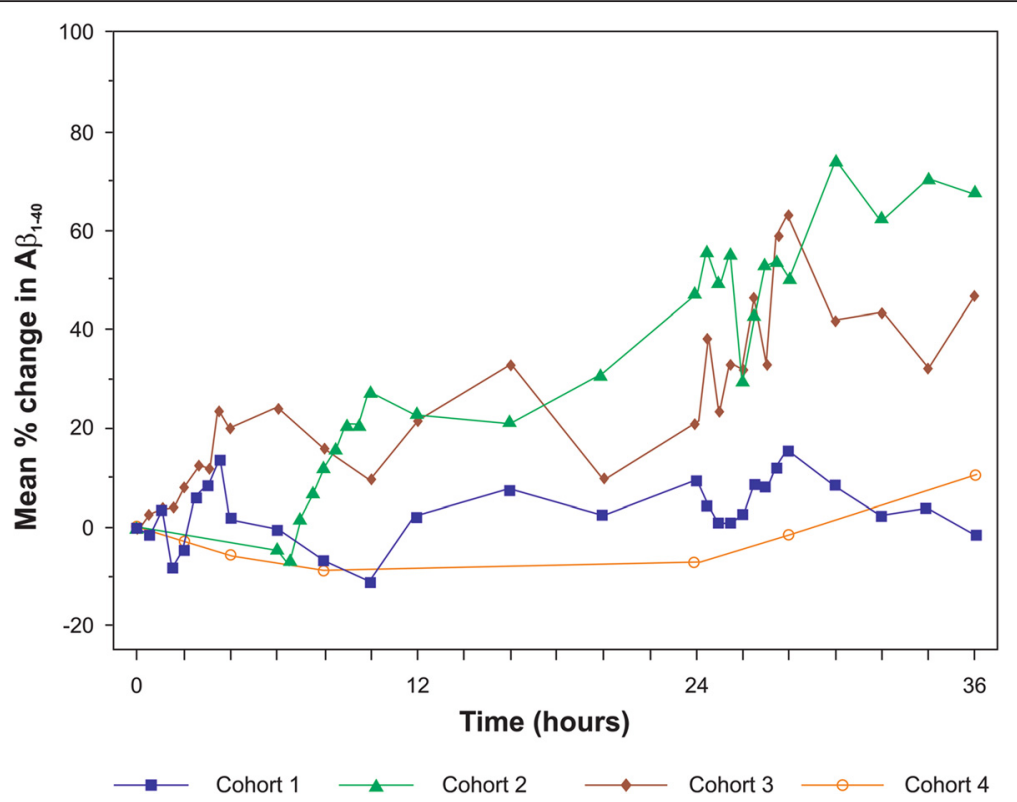

Fig. 2 Mean \% change from baseline of CSF A $\beta_{1-40}$ for all cohorts. Standard deviation is not shown for clarity of representation. Individual profiles can be found in Fig. 3. Cohort 1: immediate sampling, high frequency; cohort 2: delayed sampling, high frequency, procedure effect; cohort 3: ibuprofen, high frequency, inflammation effect; cohort 4: immediate sampling, low frequency. $A \beta$ amyloid beta

sampling. The elevation of mean $A \beta$ levels was not limited to the second period of frequent sampling (24-28 h), but started before this period and extended beyond it. Fluctuation of mean CSF $A \beta_{1-40}$ over $36 \mathrm{~h}$ was higher in cohort 2 than in cohort 1 . Increases in CSF $A \beta$ over $36 \mathrm{~h}$ were observed for all participants in cohort 2. After the 10-h time point, one participant showed increased CSF A $\beta_{1-40}$ levels of $>60 \%$ at many subsequent time points (Fig. 3b).

\section{Cohort 3: high-frequency CSF sampling scheme with immediate sampling and ibuprofen pretreatment}

Mean CSF $A \beta_{1-40}$ levels in this cohort showed more fluctuation over $36 \mathrm{~h}$ than that observed for cohort 1 (Fig. 2). Similarly to cohorts 1 and 2, CSF A $\beta$ levels increased during periods of frequent sampling. Similar to cohort 2, the CSF A $\beta$ levels remained elevated after the first period of frequent sampling. During the first period of frequent sampling $(0-4 \mathrm{~h})$, all participants, except one who showed the earliest increases in CSF A $\beta$ levels, had increased levels between 3 and $4 \mathrm{~h}$. One participant showed an increase in CSF $A \beta_{1-40}$ levels $>60 \%$ (after $12 \mathrm{~h}$ ) and $>100 \%$ (after $25 \mathrm{~h}$ ) (Fig. 3c).

Several studies support $\gamma$-secretase modulator activity of ibuprofen $[20,21]$. Mean baseline CSF $A \beta_{1-42}$ levels were not significantly reduced in the ibuprofentreated cohort versus the other cohorts (Additional file 2: Figure $\mathrm{S} 1 ; p=0.26, F$ test), suggesting that $800 \mathrm{mg}$ ibuprofen did not lower baseline CSF $A \beta_{1-42}$ levels.

\section{Cohort 4: low-frequency CSF sampling scheme with immediate sampling}

Mean CSF $A \beta_{1-40}$ levels over $36 \mathrm{~h}$ were more stable in this cohort versus the high-frequency sampling schemes (Fig. 2). Only one participant showed a pronounced increase in the CSF $A \beta_{1-40}$ levels, observed after the 28-h time point (Fig. $3 \mathrm{~d}$ ).

For all cohorts, $A \beta_{1-37}, A \beta_{1-38}$, and $A \beta_{1-42}$ were measured in addition to $A \beta_{1-40}$ and similar profiles were noted for mean change in $A \beta$ levels versus baseline (data not shown). The $A \beta$ ratios showed lower intrasubject variability than that observed for individual peptides over time, suggesting that the effect of different sampling schemes on CSF $A \beta$ levels was similar for $A \beta_{1-37}$, $\mathrm{A} \beta_{1-38}, \mathrm{~A} \beta_{1-40}$, and $\mathrm{A} \beta_{1-42}$.

\section{Possible sources of intersubject variability}

Intersubject variability was noted in all cohorts relating to increase in CSF $A \beta$ levels over time. No correlation was observed between increases in CSF A $\beta$ levels and sex, age, APOE \&4 status, MMSE score, CSF sampling or sample processing issues, or baseline levels of $\mathrm{A} \beta_{1-42}, \mathrm{P}-\mathrm{tau}_{181 \mathrm{P}}$ and $\mathrm{T}$-tau (Additional file 3: Table S1).

A trend for a higher incidence of headache (10/15 $(66.7 \%))$ was observed in participants with an $A \beta$ increase of $>25 \%$ versus baseline compared with participants with an $\mathrm{A} \beta$ increase $<25 \%(2 / 8(25 \%))$ (Fisher's exact test: $p=0.089$ ). 

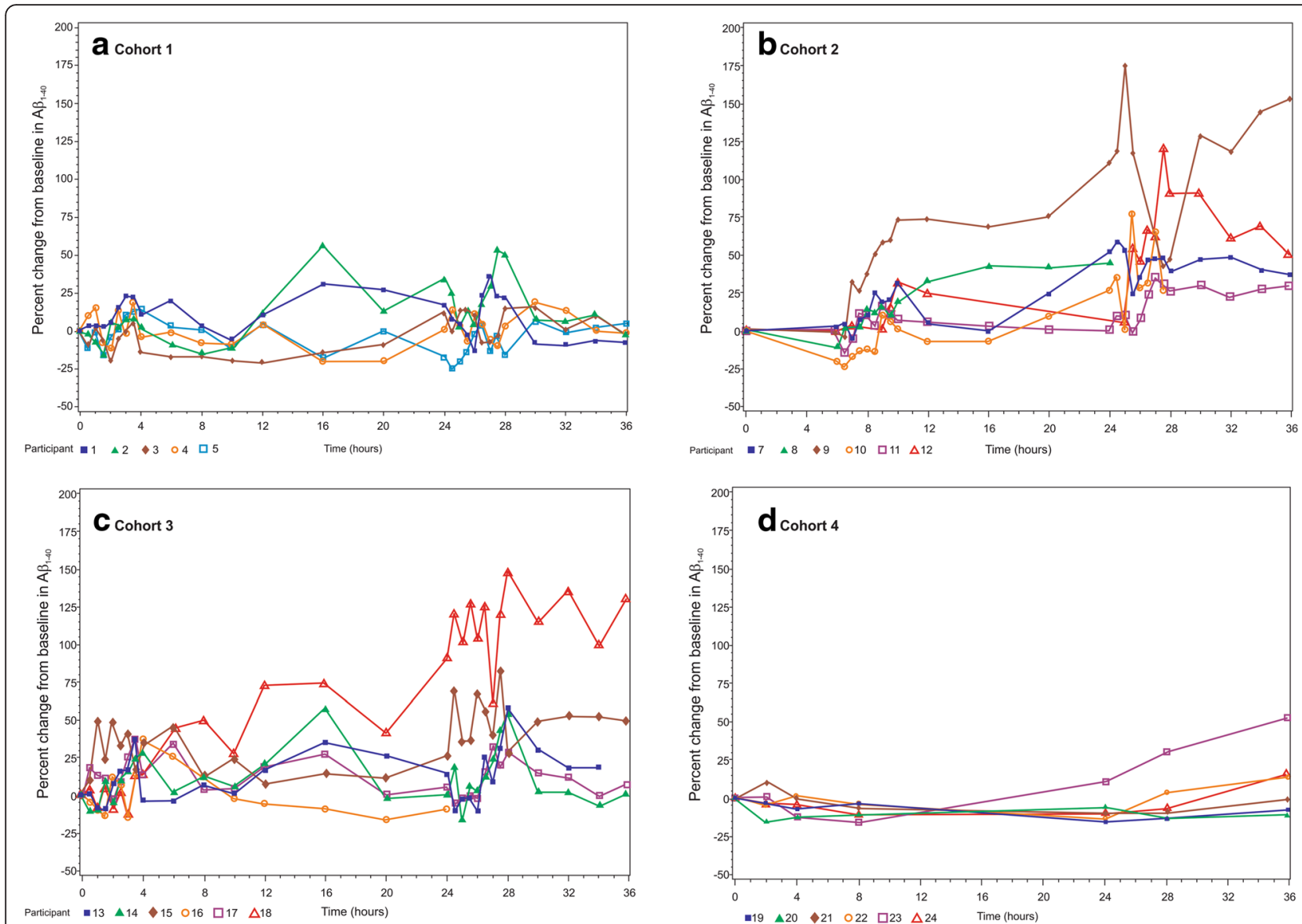

Fig. 3 Individual CSF $A \beta_{1-40}$ profiles for all participants per cohort. a Cohort 1: immediate sampling, high frequency. b Cohort 2: delayed sampling, high frequency, procedure effect. c Cohort 3: ibuprofen, high frequency, inflammation effect. d Cohort 4: immediate sampling, low frequency. $A P O E$ \&4 carriers are indicated in red with a square or diamond symbol. Cohort 1: only five participants are shown; one participant (participant 6) did not have a baseline sample available. $A \beta$ amyloid beta (Color figure online)

\section{Safety and tolerability of CSF sampling procedures}

Nineteen (79.2 \%) participants experienced at least one AE, which was mild or moderate in severity, and the majority of events were resolved at follow-up. Incidence of AEs was not significantly different across cohorts $(p=0.11$, Fisher's exact test). Headache $(n=12$ $(50.0 \%))$, back pain $(n=7(29.2 \%))$, and catheter-site pain $(n=3(12.5 \%))$ were most frequently reported, and were possibly related to the sampling procedure (Table 2). There was only one occurrence $(4.2 \%)$ of postdural puncture headache. No clinically relevant changes in vital signs, ECG, or laboratory measurements were noted. There were no signs of inflammation as indicated by clinical laboratory measurements.

\section{Discussion}

CSF A $\beta$ concentrations were shown to be stable over months to years in longitudinally collected samples with isolated lumbar punctures [22, 23]. However, the intrasubject CSF $A \beta_{1-40}$ and $A \beta_{1-42}$ levels varied largely and tended to rise over $36 \mathrm{~h}$ after hourly sampling via an intrathecal catheter in healthy participants [5]. Consistent with other studies, an increase in CSF A $\beta$ levels following serial samplings was noted in our study $[3,4,6$, $7,9]$. Factors such as frequency of sampling and sample volume [4], diurnal effects $[5,7,8]$, catheter interaction with CSF $A \beta$ at the first sampling time points, and activity $[5,7]$ were suggested to be related to this observed increase. However, further studies to delineate the exact mechanisms are needed.

We evaluated the effect of different CSF sampling protocols on CSF A $\beta$ levels in an older population to reflect the age range of the target population for AD. Earlier studies mainly describe CSF A $\beta$ changes for younger healthy participants. A study comparing data from older healthy controls and $\mathrm{AD}$ patients showed that the variation in CSF measures of $A \beta_{1-40}, A \beta_{1-42}, T$-tau, and P-tau ${ }_{181 \mathrm{P}}$ was comparable [8]. On the other hand, hour-to-hour fluctuations in CSF biomarkers were lower in older healthy controls and $\mathrm{AD}$ patients than in young participants [5], 
Table 2 Incidence of adverse events occurring during study (starting after catheter insertion)

\begin{tabular}{|c|c|c|c|c|c|}
\hline & $\begin{array}{l}\text { Cohort } 1 \\
(n=6)\end{array}$ & $\begin{array}{l}\text { Cohort } 2 \\
(n=6)\end{array}$ & $\begin{array}{l}\text { Cohort } 3 \\
(n=6)\end{array}$ & $\begin{array}{l}\text { Cohort } 4 \\
(n=6)\end{array}$ & $\begin{array}{l}\text { Total } \\
(N=24)\end{array}$ \\
\hline Participants with one or more adverse event & $6(100.0)$ & $6(100.0)$ & $3(50.0)$ & $4(66.7)$ & $19(79.2)$ \\
\hline Headache & $3(50.0)$ & $5(83.3)$ & $2(33.3)$ & $2(33.3)$ & $12(50.0)$ \\
\hline Back pain & $1(16.7)$ & $2(33.3)$ & $1(16.7)$ & $3(50.0)$ & $7(29.2)$ \\
\hline Catheter site pain & $2(33.3)$ & 0 & $1(16.7)$ & 0 & $3(12.5)$ \\
\hline Dizziness postural & 0 & $1(16.7)$ & 0 & $1(16.7)$ & $2(8.3)$ \\
\hline Hypoesthesia & $1(16.7)$ & 0 & $1(16.7)$ & 0 & $2(8.3)$ \\
\hline Pain in extremity & $1(16.7)$ & 0 & $1(16.7)$ & 0 & $2(8.3)$ \\
\hline Nausea & 0 & $1(16.7)$ & $1(16.7)$ & 0 & $2(8.3)$ \\
\hline Musculoskeletal stiffness & 0 & $1(16.7)$ & 0 & 0 & $1(4.2)$ \\
\hline Neck pain & 0 & 0 & $1(16.7)$ & 0 & $1(4.2)$ \\
\hline Gastroesophageal reflux disease & 0 & 0 & $1(16.7)$ & 0 & $1(4.2)$ \\
\hline Regurgitation & 0 & 0 & 0 & $1(16.7)$ & $1(4.2)$ \\
\hline Toothache & 0 & 0 & 0 & $1(16.7)$ & $1(4.2)$ \\
\hline Dermatophytosis & 0 & 0 & $1(16.7)$ & 0 & $1(4.2)$ \\
\hline Sinusitis & 0 & 0 & 0 & $1(16.7)$ & $1(4.2)$ \\
\hline Post lumbar puncture syndrome & 0 & $1(16.7)$ & 0 & 0 & $1(4.2)$ \\
\hline Pruritus & $1(16.7)$ & 0 & 0 & 0 & $1(4.2)$ \\
\hline Flushing & 0 & $1(16.7)$ & 0 & 0 & $1(4.2)$ \\
\hline
\end{tabular}

Data shown as $n$ (\%). Cohort 1: immediate sampling, high frequency; cohort 2: delayed sampling, high frequency, procedure effect; cohort 3: ibuprofen, high frequency, inflammation effect; cohort 4: immediate sampling, low frequency

The laboratory parameters (clinical chemistry, hematology, urinalysis) were evaluated at screening, on days -1 and 2 , and during follow-up (days 7-14 after removal of the spinal catheter). The vital signs and resting 12-lead ECGs were evaluated at screening, on days $-1,1$, and 2, and during follow-up. The participants were physically and neurologically examined at screening, on days 1 and 3, and in follow-up. Fundoscopy was performed at screening to exclude intracranial pressure

underscoring the importance of investigating these changes in the target population.

Our results corroborated earlier findings indicating a higher increase in CSF A $\beta$ levels with protocols using high sampling frequency compared with protocols using low sampling frequency [4]. The increases in $A \beta$ levels relative to baseline were more prominent during highfrequency sampling (every $30 \mathrm{~min}$ ) in cohorts $1-3$, while the $\mathrm{A} \beta$ levels were relatively stable in samples drawn every $4 \mathrm{~h}$. Although the first period of frequent sampling occurs at a different time during the day for cohort 2 compared with cohorts 1 and 3, clear mean increases in CSF A $\beta$ levels were seen in high-frequency sampling periods for all cohorts. Together with the fact that the average $A \beta$ levels did not return to baseline after $24 \mathrm{~h}$, this suggests that the observed increase in our study could not be explained solely by a diurnal effect.

Interestingly, $A \beta$ levels at baseline and $6 \mathrm{~h}$ post catheterization were similar in cohort 2 , suggesting that the increase in $A \beta$ levels was not related to the catheter insertion procedure. Furthermore, precatheterization administration of ibuprofen did not impact the increased CSF A $\beta$ levels. These findings indicate that the increase in CSF A $\beta$ levels was not related to the procedure of catheterization itself, including any kind of induced inflammatory reaction. Together, these data support the hypothesis that the intense sampling frequency applied in these cohorts probably impacted CSF A $\beta$ levels.

After the interim review of the data from cohorts 1-3, it was hypothesized that a lower CSF sampling frequency over $36 \mathrm{~h}$ may result in more stable $\mathrm{A} \beta$ profiles. An additional cohort (cohort 4) of healthy older participants was included to assess an alternative low-frequency CSF sampling scheme. This sampling scheme, with a lower total volume of CSF sampled, resulted in more stable mean CSF A $\beta$ levels over $36 \mathrm{~h}$ versus high-frequency sampling schemes. These data support the hypothesis that the increase in CSF $\mathrm{A} \beta$ levels compared with baseline level is probably related to either CSF sampling frequency or volume, or both. The underlying mechanism of the rise in CSF A $\beta$ concentrations after frequent CSF sampling is unknown. Various stimuli, including stress and sleep deprivation, could increase brain $\mathrm{A} \beta$ levels, possibly through modulation of neuronal activity [24]. Moreover, frequent sampling in the lumbar region might alter a possible rostral-caudal $\mathrm{A} \beta$ gradient from ventricular to lumbar CSF by induction of redistribution of 
CSF to the lumbar region [4]. The disease state may also influence the CSF A $\beta$ levels and thus studies in AD patients are warranted [8].

One out of six participants in cohort 4 demonstrated a rise in CSF A $\beta$ levels after $24 \mathrm{~h}$ post catheterization. This increase does not seem to be related to gender, age, race, APOE status, MMSE score, or adverse events (Additional file 3: Table S1). As such, a possible explanation for this finding is currently unclear, but might for example include a difference in activity level of this participant. Only one participant in cohort 4 had an $A \beta_{1-42}$ concentration slightly below the threshold concentration, excluding the possibility that greater amyloid deposition in cohort 4 would be responsible for the observed stability of CSF A $\beta$ concentrations over time in this cohort [25].

\section{Conclusions}

CSF A $\beta$ levels were substantially affected by CSF sampling frequency and/or sampling volume. An optimized sampling protocol with lower CSF sampling frequency and volume resulted in more stable $A \beta$ profiles. In future clinical studies with $A \beta$-targeting experimental drugs, this protocol would lead to a better estimation of drug effects on $A \beta$ levels after continuous CSF sampling and thus lower the required sample size. The described protocols for continuous CSF sampling were well tolerated with no clinically important safety concerns in healthy older participants. These results substantiate the potential of CSF $A \beta$ as a pharmacodynamic biomarker using frequent CSF sampling to assess evidence of target engagement and pharmacological activity of $\mathrm{A} \beta$-targeting compounds.

\section{Statistical analysis}

JB was the project statistician (Janssen Research \& Development, LLC, Raritan, NJ, USA).

\section{Ethics approval}

Commissie voor Medische Ethiek, ZNA, Antwerp, Belgium (Institutional Review Board) approved the study.

\section{Additional files}

$$
\begin{aligned}
& \text { Additional file 1: Details the inclusion and exclusion criteria of the } \\
& \text { study. (PDF } 7 \mathrm{~kb} \text { ) }
\end{aligned}
$$

Additional file 2: Figure S1 showing scatter plots representing measures of individual participants for baseline concentrations of CSF $A \beta_{1-42}, P-t_{10 u} 18$, and T-tau per cohort. (PDF $131 \mathrm{~kb}$ )

Additional file 3: Table S1 presenting an overview of individual participant characteristics at baseline. (PDF $97 \mathrm{~kb}$ )

\section{Abbreviations}

AD: Alzheimer's disease; AE: adverse event; APOE: apolipoprotein E; $A B$ : amyloid beta; BMI: body mass index; CSF: cerebrospinal fluid; CV: coefficient of variation; ELISA: enzyme-linked immunosorbent assay;
GCP: Good Clinical Practices; ICH: International Conference on Harmonization; MMSE: Mini-Mental State Examination; MSD: Meso Scale Discovery; P-tau: phosphorylated tau; T-tau: total tau.

\section{Competing interests}

All authors, except SR, LMS, and SE, report personal fees (employment) from Janssen Research \& Development, a division of Janssen Pharmaceutica N.V., Beerse, Belgium, or Janssen Research \& Development, LLC, Raritan, NJ, USA and own stock/stock options in the company. SR reports personal fees (employment) from SGS Life Science Services, the Contract Research Organization that conducted the clinical part of the trial. SR does not own stock/options of Janssen Pharmaceutica N.V., and neither did he receive any direct payments from Janssen Pharmaceutica N.V. LMS reports consulting (biomarker analyses and interpretation) fees from Eli Lilly and Janssen Research \& Development; and consulting fees from Novartis. SE reports research funding from Janssen Pharmaceutica N.V., ADx NeuroSciences, and Lundbeck; and consulting fees from Innogenetics/Fujirebio Europe, Lundbeck, Pfizer, Novartis, UCB, Roche diagnostics, and Nutricia/Danone. No nonfinancial conflicts of interest exist for any of the authors.

\section{Authors' contributions}

JRS was involved in conception of the study, study design, collection of data, and drafting the manuscript. LVN participated in the design of the study, data collection, and drafting the manuscript. MT and BVB participated in drafting and revising the manuscript, and data collection and analysis. JS, LMS, SE, and MM were involved in collection of data and participated in drafting the manuscript. JB performed the statistical analysis and was involved in interpretation of data and revising the manuscript. SR was the principal investigator for this study, was responsible for study execution, and participated in drafting the manuscript. All authors read and approved the manuscript.

\section{Acknowledgements}

The authors acknowledge Rishabh Pandey and Pravin Bolshete (SIRO Clinpharm Pvt. Ltd) for writing assistance and Wendy Battisti (Janssen Research \& Development, LLC) for additional editorial support. They acknowledge Bart Hermans and Marianne Borgers (Janssen Research \& Development) for technical support in conduction of CSF A $\beta$ analyses. Written informed consent was obtained from the participants for publication of their individual details without disclosing their identity in this manuscript. The consent form is held by the authors' institution and is available for review by the Editor-in-Chief.

\section{Funding}

Funding support was provided by Janssen Research \& Development, a division of Janssen Pharmaceutica N.V., Beerse, Belgium.

\section{Author details \\ 'Janssen Research \& Development, a division of Janssen Pharmaceutica N.V., Beerse, Belgium. ${ }^{2}$ SGS Life Science Services, Antwerp, Belgium. ${ }^{3}$ Janssen Research \& Development, LLC, Raritan, NJ, USA. ${ }^{4}$ University of Pennsylvania Medical Center, Philadelphia, PA, USA. ${ }^{5}$ Reference Center for Biological Markers of Dementia (BIODEM), Institute Born-Bunge, University of Antwerp, Antwerp, Belgium. ${ }^{6}$ Department of Neurology and Memory Clinic, Hospital Network Antwerp (ZNA) Middelheim and Hoge Beuken, Antwerp, Belgium.}

Received: 4 December 2015 Accepted: 11 April 2016 Published online: 19 May 2016

\section{References}

1. Blennow $\mathrm{K}$, Hampel $\mathrm{H}$, Weiner M, Zetterberg H. Cerebrospinal fluid and plasma biomarkers in Alzheimer disease. Nat Rev Neurol. 2010;6:131-44.

2. Blennow K, Zetterberg H, Fagan AM. Fluid biomarkers in Alzheimer disease. Cold Spring Harb Perspect Med. 2012;2:a006221.

3. Bateman RJ, Siemers ER, Mawuenyega KG, Wen G, Browning KR, Sigurdson WC, et al. A gamma-secretase inhibitor decreases amyloid-beta production in the central nervous system. Ann Neurol. 2009;66:48-54.

4. Li J, Llano DA, Ellis T, LeBlond D, Bhathena A, Jhee SS, et al. Effect of human cerebrospinal fluid sampling frequency on amyloid-beta levels. Alzheimers Dement. 2012;8:295-303. 
5. Bateman RJ, Wen G, Morris JC, Holtzman DM. Fluctuations of CSF amyloidbeta levels: implications for a diagnostic and therapeutic biomarker. Neurology. 2007;68:666-9.

6. Forman M, Palcza J, Tseng J, Leempoels J, Ramael S, Han D, et al. The novel BACE inhibitor MK-8931 dramatically lowers cerebrospinal fluid A $\beta$ peptides in healthy subjects following single- and multiple-dose administration. Alzheimers Dement. 2012;8(4):704.

7. Kang JE, Lim MM, Bateman RJ, Lee JJ, Smyth LP, Cirrito JR, et al. Amyloidbeta dynamics are regulated by orexin and the sleep-wake cycle. Science. 2009;326:1005-7.

8. Slats D, Claassen JA, Spies PE, Borm G, Besse KT, van Aalst W, et al. Hourly variability of cerebrospinal fluid biomarkers in Alzheimer's disease subjects and healthy older volunteers. Neurobiol Aging. 2012;33:831. e1-9.

9. Tong G, Castaneda L, Wang JS, Sverdlov O, Huang SP, Slemmon R, et al. Effects of single doses of avagacestat (BMS-708163) on cerebrospinal fluid Abeta levels in healthy young men. Clin Drug Investig. 2012;32:761-9.

10. Verwey NA, van der Flier WM, Blennow K, Clark C, Sokolow S, De Deyn PP, et al. A worldwide multicentre comparison of assays for cerebrospinal fluid biomarkers in Alzheimer's disease. Ann Clin Biochem. 2009;46:235-40.

11. Mattsson N, Andreasson U, Persson S, Carrillo MC, Collins S, Chalbot S, et al. CSF biomarker variability in the Alzheimer's Association quality control program. Alzheimers Dement. 2013;9:251-61.

12. Florkowski CM. Preanalytical variables and Alzheimer disease biomarker concentrations in cerebrospinal fluid. Clin Chem. 2015;61:686-88.

13. Le Bastard N, De Deyn PP, Engelborghs S. Importance and impact of preanalytical variables on Alzheimer disease biomarker concentrations in cerebrospinal fluid. Clin Chem. 2015;61:734-43.

14. Van Broeck B, Borgers M, Meulders G, Hermans B, Dhuyvetter D, Borghys H, et al. Simultaneous evaluation of $A \beta_{1-37 / 38 / 40 / 42}$ levels after treatment with secretase inhibitors and modulators using a novel immunoassay. Neurodegener Dis. 2013;11.

15. Struyfs $H$, Van Broeck B, Timmers M, Fransen E, Sleegers $K$, Van Broeckhoven $C$, et al. Diagnostic accuracy of CSF amyloid- $\beta$ isoforms for early and differential dementia diagnosis. J Alzheimers Dis. 2015;45:813-22.

16. Mercken M, Brepoels E, De Jongh M, Laenen W, Raeymaekers P, Geerts H, et al. Specific ELISA systems for the detection of endogenous human and rodent $A \beta_{40}$ and $A \beta_{42}$. Neurobiol Aging. 2000;21:S41.

17. Shaw LM, Vanderstichele H, Knapik-Czajka M, Figurski M, Coart E, Blennow K, et al. Qualification of the analytical and clinical performance of CSF biomarker analyses in ADNI. Acta Neuropathol. 2011;121:597-609.

18. Dean R, Shaw LM, Waligorska TW, Korecka M, Figurski M, Trojanowski J, et al. Inclusion of patients with Alzheimer's disease pathology in solanezumab expedition 3 using florbetapir PET imaging or INNO-BIA AlzBio3 CSF A $\beta_{1-42}$. Alzheimers Dement. 2014:10:P811.

19. Favis R, Gerry NP, Cheng YW, Barany F. Applications of the universal DNA microarray in molecular medicine. Methods Mol Med. 2005;114:2-58.

20. Imbimbo BP, Giardina GA. $y$-secretase inhibitors and modulators for the treatment of Alzheimer's disease: disappointments and hopes. Curr Top Med Chem. 2011;11:1555-70.

21. Weggen S, Eriksen JL, Das P, Sagi SA, Wang R, Pietrzik CU, et al. A subset of NSAIDs lower amyloidogenic $A \beta_{42}$ independently of cyclooxygenase activity. Nature. 2001;414:212-16.

22. Blennow K, Zetterberg H, Minthon L, Lannfelt L, Strid S, Annas P, et al. Longitudinal stability of CSF biomarkers in Alzheimer's disease. Neurosci Lett. 2007:419:18-22.

23. Zetterberg H, Pedersen $M$, Lind $K$, Svensson M, Rolstad S, Eckerström C, et al. Intra-individual stability of CSF biomarkers for Alzheimer's disease over two years. J Alzheimers Dis. 2007;12:255-60.

24. Bissette G. Does Alzheimer's disease result from attempts at repair or protection after transient stress? J Alzheimers Dis. 2009;18:371-80.

25. Huang Y, Potter R, Sigurdson W, Santacruz A, Shih S, Ju YE, et al. Effects of age and amyloid deposition on A $\beta$ dynamics in the human CNS. Arch Neurol. 2012;69:51-8.

\section{Submit your next manuscript to BioMed Central and we will help you at every step:}

- We accept pre-submission inquiries

- Our selector tool helps you to find the most relevant journal

- We provide round the clock customer support

- Convenient online submission

- Thorough peer review

- Inclusion in PubMed and all major indexing services

- Maximum visibility for your research

Submit your manuscript at www.biomedcentral.com/submit
C Biomed Central 